\title{
Implicaciones de la geometría, uso del suelo y dispositivos que controlen intersecciones de un corredor vial de flujo continuo
}

\section{Implications of geometry, land use and control devices that control the intersections on a continuous flow corridor}

\section{Carlos Felipe Urazán}

Ingeniero Civil, doctor en Gestión Territorial e Infraestructura del Transporte. Docente Asociado de la Universidad de La Salle. Bogotá, Colombia.

Contacto:caurazan@unisalle.edu.co.

\section{Luisa Fernanda Garzón}

Ingeniera Civil. Investigadora de la Universidad de La Salle. Bogotá, Colombia. Contacto:luisa.g7@hotmail.com.

\section{Mireya Ardila}

Ingeniera Civil. Investigadora de la Universidad de La Salle. Bogotá, Colombia. Contacto:mardila88@gmail.com.

\section{Hugo Rondón}

Ingeniero Civil, doctor en Ingeniería con énfasis en infraestructura vial. Docente Asociado de la Universidad Distrital Francisco José de Caldas. Bogotá, Colombia. Contacto: harondonq@udistrital.edu.co.

\section{Nó́ Villegas Flóres}

Ingeniero civil, doctor en ingeniería de la construcción. Docente de la Universidad Federal de la Integración Latinoamericana - UNILA. Foz de Iguazú, Brasil. Contacto:noe.flores@unila.edu.br

\section{Fabián Augusto Lamus}

Ingeniero Civil, candidato a doctor en Ciencia y tecnología de materiales. Docente Asistente de la Universidad de La Salle. Bogotá, Colombia.

Contacto:falamus@unisalle.edu.co. 


\section{investigación}

Palabras clave: autopista norte, desarrollo urbano, intersección vial, movilidad urbana.

Key words: autopista norte highway, road intersection, urban development, urban mobility.

\section{RESUMEN}

La Autopista Norte es una arteria vial de tránsito continuo en la ciudad de Bogotá. De acuerdo con la Secretaría de Movilidad, llegan a circular de lunes a viernes cerca de 18000 vehículos por esta arteria vial. En su trayecto en el casco urbano se presentan intersecciones con vías secundarias que registran diversas particularidades. La presencia de reducción de carriles, semáforos y retornos en inmediaciones de la intersección y orejas manzana de considerable longitud, afectan el flujo vehicular circundante y por tanto los tiempos que requiere realizar los movimientos, tanto directos como a izquierda y derecha en la respectiva intersección. La observación del fenómeno de demoras invita a que la planificación de nuevos desarrollos contemple soluciones que anticipen adecuadamente el aumento de flujo vehicular para permitir tiempos adecuados al emplear las intersecciones, especialmente en horas punta.

\section{ABSTRACT}

The Autopista Norte Highway is a continuous traffic artery way in Bogota city. According to "Mobility Secretary", this main road could reach a traffic about eighteen thousand vehicles. On the urban sector the intersections with secondary roads exposes various particularities. The presence of reducing lanes, traffic lights and return ways near the intersection and considerable length of "orejas de manzana", could affected the vehicle traffic flow. As a consequence, could rises the time required to allow the vehicle to move right, left and straight in the respective intersections. Therefore, the delay time phenomenon invites to planning new developments that could increase flow traffic performance in the respective vehicles movements: right, left and straight in the intersections. As result, it would reduce the time that the vehicles could spend in the intersections, especially at peak hour.

\section{INTRODUCCIÓN}

Bogotá D.C., es una ciudad que cuenta, para el 2013, con una población aproximada de 7.675.000 habitantes [1]. Su condición de ciudad metropolitana hace vital la presencia de vías arterias como son: la Carrera Séptima, la Avenida Caracas y la Transversal Norte Quito Sur (NQS). A pesar de la existencia de los corredores mencionados, el flujo vehicular en hora punta se ve afectado por la incapacidad operativa de los mismos corredores para atender la demanda vehicular actual, generando situaciones de congestionamiento vehicular, afectando la calidad de vida de sus habitantes y volviendo poco eficaz la economía propia y de la región [2]. 


\section{investigación}

la calle 170. La importancia de las intersecciones radica en que el nivel de servicio de la vía principal se ve afectado pues es recurrente la necesidad de disminuir la velocidad y, en algunas ocasiones, se presentan importantes esperas debido a la semaforización obligada para realizar alguno de los movimientos: directo, giro a izquierda, giro a derecha [4]. Ha de recordarse que las intersecciones presentes en la Autopista Norte son de tipo a desnivel con rampas, pues las intersecciones a nivel implicarían un semáforo o una glorieta lo que generaría un aumento considerable de demoras, afectando negativamente el nivel de servicio de la intersección. Las demoras serían causadas principalmente por la obligada reducción de la velocidad y, si la intensidad de tráfico es elevada, puede ser preciso esperar durante algún tiempo antes de poder atravesar la intersección [4]; lo que generaría mayor demora y, según la situación, posible congestionamiento vial. La investigación llevada a cabo establece los efectos que la geometría vial, el uso del suelo y los dispositivos de control de tránsito, tienen en los tiempos de giro en las intersecciones elevadas en una vía arteria.

En la literatura especializada se encuentran diversos estudios sobre planteamientos de intersecciones de flujo continuo, como [5 y 6], pero no se hallaron evaluaciones respecto a la influencia de elementos como retornos, dispositivos de control y la presencia de orejas manzana en el flujo vehicular. Por otro lado, se ha evidenciado situaciones de demora y represamiento del tráfico en la Autopista Norte, que implican demoras hasta de 40 minutos, hechos registrados recientemente en beneficio de alertar la urgente necesidad de descongestionar el corredor vial [ 7 y 8 ].

\section{METODOLOGÍA}

El trabajo se realizó mediante la toma de información primaria de campo en la que se aforó el tráfico vehicular registrando: el flujo vehicular y los tiem- pos de recorrido promedio en todas las entradas y salidas a la autopista por las intersecciones analizadas (calle 100, calle 116, calle 127 , calle 134 , calle 153, calle 170). El estudio se concentró en horas punta, de 6:00 a.m 8:00 a.m. y de 5:30 a 8:00 p.m. en días laborales. Los aforos fueron realizados tres veces para cada movimiento, mediante captura de video digital. Una vez registrada la información en video, se codificaron numéricamente cada uno de los movimientos de las intersecciones para facilitar su identificación en el análisis. Si bien los videos permitieron realizar los conteos y clasificación vehicular, los tiempos de recorrido para cada movimiento se obtuvieron por desplazamiento de los investigadores en vehículo particular.

La información de las longitudes recorridas en cada movimiento se obtuvo por intermedio del software online Google Earth (figura 1). Paso posterior, se realizó un análisis comparado de los tiempos de recorrido en los movimientos: izquier$\mathrm{da}$, directo y derecha, en cada una de las intersecciones, aplicado a todos los movimientos. Las diferencias significativas encontradas en los tiempos fueron discriminados a partir de la geometría de la ruta del movimiento, su longitud y continuidad, debida a intersecciones o dispositivos de control de tránsito como semáforos. Adicionalmente, la actividad del suelo circundante ayudó a determinar el escenario de tránsito y la presencia de dispositivos de control.

\section{FLUJO VEHICULAR Y TIEMPOS DE RECORRIDO}

De la tabla 1 a la tabla 6 se presentan los registros promedio obtenidos para el flujo vehicular y los tiempos de recorrido en los movimientos: izquierda, directo y derecha, por intersección y para cada movimiento.

Para el análisis de estos resultados, se dividieron los movimientos en directos, a derecha y a

Implicaciones de la geometría, uso del suelo y dispositivos que controlen intersecciones de un corredor vial de flujo continuo 


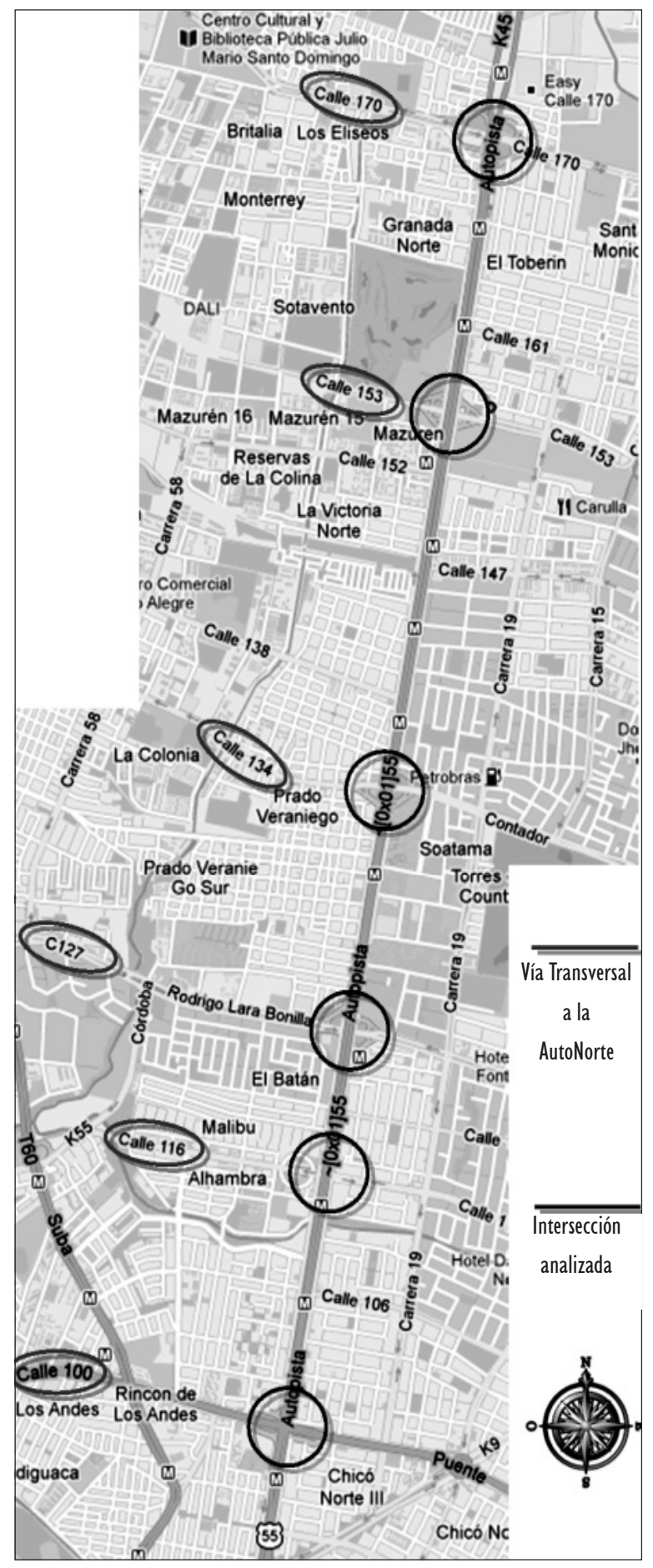

Figura 1. Zona de análisis. Autopista Norte y vías transversales

Fuente: elaboración propia a partir de Google Maps (noviembre 2012).
Tabla 1. Resultados intersección calle 100

\begin{tabular}{|c|l|c|c|}
\hline Movimiento & Sentido & $\begin{array}{c}\text { Tiempo de } \\
\text { recorrido }\end{array}$ & $\begin{array}{c}\text { Flujo } \\
\text { vehicular } \\
\text { (veh/hora) }\end{array}$ \\
\hline 1 & S - N & $10^{\prime \prime}$ & 1000 \\
\hline 2 & S - OCC & $1^{\prime} 11^{\prime \prime}$ & 764 \\
\hline 3 & OR - OCC & $38^{\prime \prime}$ & 867 \\
\hline 4 & OR - S & $4^{\prime} 11^{\prime \prime}$ & 242 \\
\hline 5 & N - S & $17^{\prime \prime}$ & 519 \\
\hline 6 & N - OR & $3^{\prime} 57^{\prime \prime}$ & 823 \\
\hline 7 & OCC - OR & $38^{\prime \prime}$ & 445 \\
\hline 8 & OCC - N & $5{ }^{\prime} 28^{\prime \prime}$ & 812 \\
\hline 9 & OR - N & $23^{\prime \prime}$ & 488 \\
\hline 10 & S - OR & $20^{\prime \prime}$ & 295 \\
\hline 11 & N - OCC & $49^{\prime \prime}$ & 504 \\
\hline 12 & OCC - S & $15^{\prime \prime}$ & 431 \\
\hline
\end{tabular}

Fuente: elaboración propia

Tabla 2. Resultados intersección calle 116

\begin{tabular}{|c|c|c|c|}
\hline Movimiento & Sentido & $\begin{array}{l}\text { Tiempo de } \\
\text { recorrido }\end{array}$ & $\begin{array}{c}\text { Flujo } \\
\text { vehicular } \\
\text { (veh/hora) }\end{array}$ \\
\hline 1 & $S-N$ & $46 "$ & 1819 \\
\hline 2 & S - OCC & 4' 55" & 528 \\
\hline 3 & OR - OCC & $32 "$ & 1719 \\
\hline 4 & OR - S & 3' 30" & 588 \\
\hline 5 & $N-S$ & 1'38'" & 1836 \\
\hline 6 & $\mathrm{~N}-\mathrm{OR}$ & 6' 42" & 643 \\
\hline 7 & OCC - OR & 3' 56" & 1719 \\
\hline 8 & $\mathrm{OCC}-\mathrm{N}$ & 8' 29"' & 638 \\
\hline 9 & OR - N & $34 "$ & 505 \\
\hline 10 & S - OR & $34 "$ & 591 \\
\hline 11 & $\mathrm{~N}-\mathrm{OCC}$ & $17 "$ & 271 \\
\hline 12 & OCC - S & $40 "$ & 280 \\
\hline 13 & OR - OR & 3'07"' & 554 \\
\hline 14 & OR - OCC & $36 "$ & 65 \\
\hline 15 & $\mathrm{~N}-\mathrm{OR}$ & 4'03" & 564 \\
\hline
\end{tabular}

Fuente: elaboración propia 


\section{investigación}

Tabla 3. Resultados intersección calle 127

\begin{tabular}{|c|l|c|c|}
\hline Movimiento & Sentido & $\begin{array}{c}\text { Tiempo de } \\
\text { recorrido }\end{array}$ & $\begin{array}{c}\text { Flujo } \\
\text { vehicular } \\
\text { (veh/hora) }\end{array}$ \\
\hline 1 & S - N & $20^{\prime \prime}$ & 525 \\
\hline 2 & S - OCC & $1^{\prime} 08^{\prime \prime}$ & 983 \\
\hline 3 & OR - OCC & $29^{\prime \prime}$ & 696 \\
\hline 4 & OR - S & $2^{\prime} 06^{\prime \prime}$ & 688 \\
\hline 5 & N - S & $1^{\prime} 28^{\prime \prime}$ & 1906 \\
\hline 6 & N - OR & $6^{\prime}$ & 573 \\
\hline 7 & OCC - OR & $1^{\prime} 23^{\prime \prime}$ & 864 \\
\hline 8 & OCC - N & $1^{\prime} 13^{\prime \prime}$ & 721 \\
\hline 9 & OR - N & $14^{\prime \prime}$ & 403 \\
\hline 10 & S - OR & $27^{\prime \prime}$ & 128 \\
\hline 11 & N - OCC & $15^{\prime \prime}$ & 213 \\
\hline 12 & OCC - S & $11^{\prime \prime}$ & 357 \\
\hline
\end{tabular}

Fuente: elaboración propia

Tabla 4. Resultados intersección calle 134

\begin{tabular}{|c|l|c|c|}
\hline Movimiento & Sentido & $\begin{array}{c}\text { Tiempo de } \\
\text { recorrido }\end{array}$ & $\begin{array}{c}\text { Flujo } \\
\text { vehicular } \\
\text { (veh/hora) }\end{array}$ \\
\hline 1 & S - N & $44^{\prime \prime}$ & 1377 \\
\hline 2 & S - OCC & $3^{\prime} 09^{\prime \prime}$ & 648 \\
\hline 3 & OR - OCC & $2^{\prime} 03^{\prime \prime}$ & 784 \\
\hline 4 & OR - S & $6^{\prime} 01^{\prime \prime}$ & 1052 \\
\hline 5 & N - S & $54^{\prime \prime}$ & 1829 \\
\hline 6 & N - OR & $3^{\prime} 39^{\prime \prime}$ & 496 \\
\hline 7 & OCC - OR & $1^{\prime} 12^{\prime \prime}$ & 1141 \\
\hline 8 & OCC - N & $5^{\prime} 31^{\prime}$ & 955 \\
\hline 9 & OR - N & $29^{\prime \prime}$ & 195 \\
\hline 10 & S - OR & $16^{\prime \prime}$ & 508 \\
\hline 11 & N - OCC & $1^{\prime} 08^{\prime \prime}$ & 57 \\
\hline 12 & OCC - S & $18^{\prime \prime}$ & 375 \\
\hline
\end{tabular}

Fuente: elaboración propia
Tabla 5. Resultados intersección calle 153

\begin{tabular}{|c|l|c|c|}
\hline Movimiento & Sentido & $\begin{array}{c}\text { Tiempo de } \\
\text { recorrido }\end{array}$ & $\begin{array}{c}\text { Flujo } \\
\text { vehicular } \\
\text { (veh/hora) }\end{array}$ \\
\hline 1 & S - N & $54^{\prime \prime}$ & 868 \\
\hline 2 & S - OCC & $5^{\prime}$ '08' & 985 \\
\hline 3 & OR - OCC & $2^{\prime} 12^{\prime \prime}$ & 865 \\
\hline 4 & OR - S & $3^{\prime} 24^{\prime \prime}$ & 1008 \\
\hline 5 & N - S & $13^{\prime \prime}$ & 1148 \\
\hline 6 & N - OR & $11^{\prime \prime}$ & 565 \\
\hline 7 & OCC - OR & $29^{\prime \prime}$ & 325 \\
\hline 8 & OCC - N & $45^{\prime \prime}$ & 380 \\
\hline 9 & OR - N & $9{ }^{\prime \prime}$ & 192 \\
\hline 10 & S - OR & $166^{\prime \prime}$ & 255 \\
\hline 11 & N - OCC & $366^{\prime \prime}$ & 84 \\
\hline 12 & OCC - S & $26^{\prime \prime}$ & 240 \\
\hline
\end{tabular}

Fuente: elaboración propia

Tabla 6. Resultados intersección calle 170

\begin{tabular}{|c|c|c|c|}
\hline Movimiento & Sentido & $\begin{array}{c}\text { Tiempo de } \\
\text { recorrido }\end{array}$ & $\begin{array}{c}\text { Flujo } \\
\text { vehicular } \\
\text { (veh/hora) }\end{array}$ \\
\hline 1 & S - N & $1^{\prime} 17^{\prime \prime}$ & 1034 \\
\hline 2 & S - OCC & $2^{\prime} 14^{\prime \prime}$ & 1152 \\
\hline 3 & OR - OCC & $28^{\prime \prime}$ & 953 \\
\hline 4 & OR - S & $4^{\prime} 27^{\prime \prime}$ & 469 \\
\hline 5 & N - S & $30^{\prime \prime}$ & 765 \\
\hline 6 & N - OR & $1^{\prime} 51^{\prime \prime}$ & 1111 \\
\hline 7 & OCC - OR & $36^{\prime \prime}$ & 569 \\
\hline 8 & OCC - N & $1^{\prime} 12^{\prime \prime}$ & 1116 \\
\hline 9 & OR - N & $16^{\prime \prime}$ & 665 \\
\hline 10 & S - OR & $27^{\prime \prime}$ & 412 \\
\hline 11 & N - OCC & $39^{\prime \prime}$ & 565 \\
\hline 12 & OCC - S & $26^{\prime \prime}$ & 351 \\
\hline
\end{tabular}

Fuente: elaboración propia 


\section{investigación}

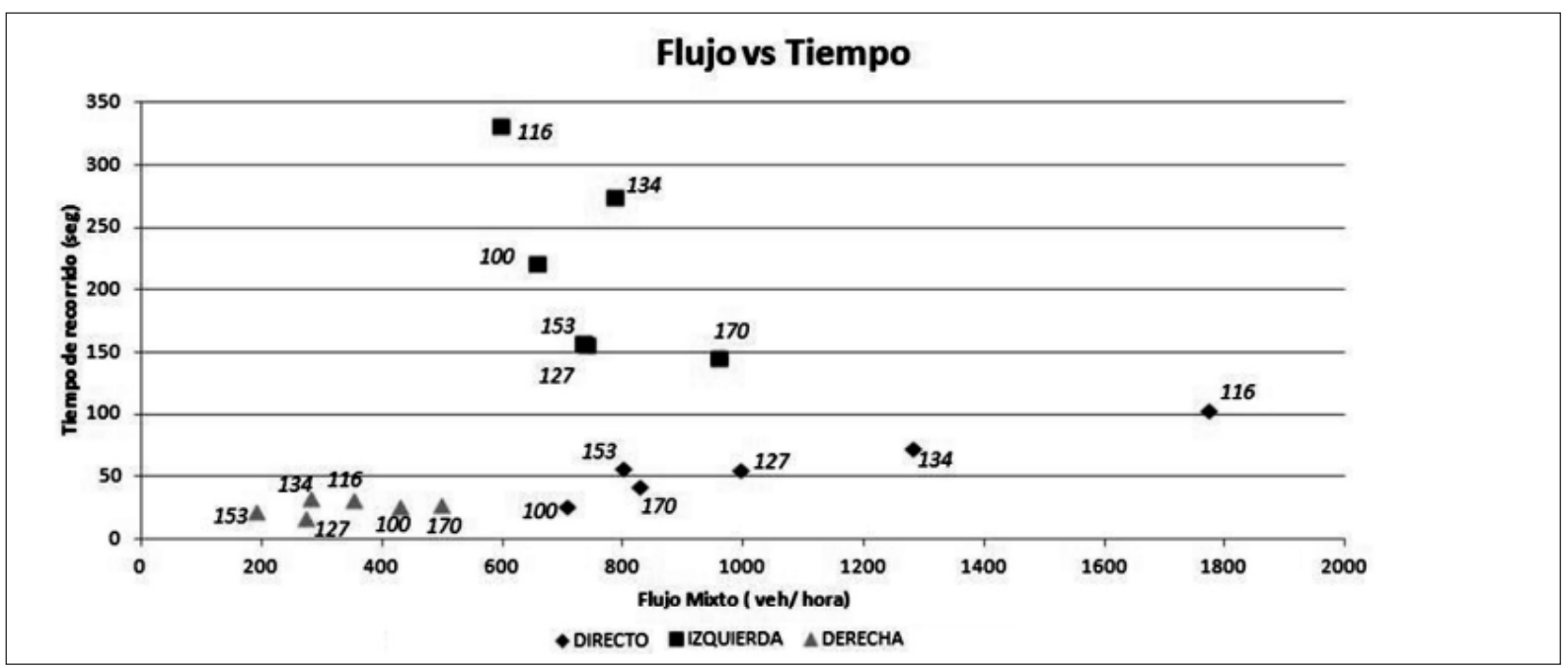

Figura 2. Flujo Vs Tiempo

Fuente: elaboración propia.

izquierda; para cada uno de los cuales se registraron: tiempos promedio de recorrido y el flujo vehicular (figuras 1 y 2).

La figura 2 pone de manifiesto que los giros a derecha son los que menor flujo vehicular presenta ( $<600 \mathrm{veh} / \mathrm{hora})$ y, por tanto, una tendencia a menores velocidades de recorrido $(<50 \mathrm{seg}$.). Por su parte, los movimientos directos y a izquierda comparten flujo vehicular en gran parte de las intersecciones (entre 600 y $1100 \mathrm{veh} /$ hora), pero los tiempos de recorrido difieren notablemente: los movimientos directos están por debajo de los 100 segundos, mientras que los movimientos a izquierda -tomando oreja y luego puente- están entre los 150 y 325 segundos. Lo anterior, se sustenta, en principio, por la longitud a recorrer (figura 2). Cabe destacar que las intersecciones calle 116 y calle 134 son, en ese orden, las que mayor tiempo de recorrido registran, para cada uno de los tres movimientos.

La figura 3 muestra una clara diferencia en las longitudes a recorrer en los movimientos. Los movimientos a derecha presentan las menores longitudes -entre 200 y 400 metros-, seguido con longitudes levemente superiores en los movimientos directos -entre 400 y 600 metros-. Finalmente, y con longitudes superiores a las anteriores están los movimientos a izquierda con valores entre los 700 y 1000 metros. No obstante, debido a la configuración geométrica de los accesos, la intersección de la calle 116 muestra longitudes para giro a izquierda cercanos a los 1400 metros, valor atípico en la muestra del estudio.

Si bien, en principio podría pensarse que la relación entre las variables tiempo y distancia o longitud a recorrer sería: a mayor longitud mayor tiempo de recorrido, no existe una correlación definida debido a las distintas condiciones de flujo vehicular que hay en las intersecciones. Ejemplificando, las longitudes de recorrido son similares en las intersecciones 100, 127, 134, 153 y 170. No obstante, los tiempos de recorrido solo son similares en los movimientos a derecha, se distancian un poco más en los movimientos directos, y aún más en los movimientos a izquierda (figura 2). 


\section{investigación}

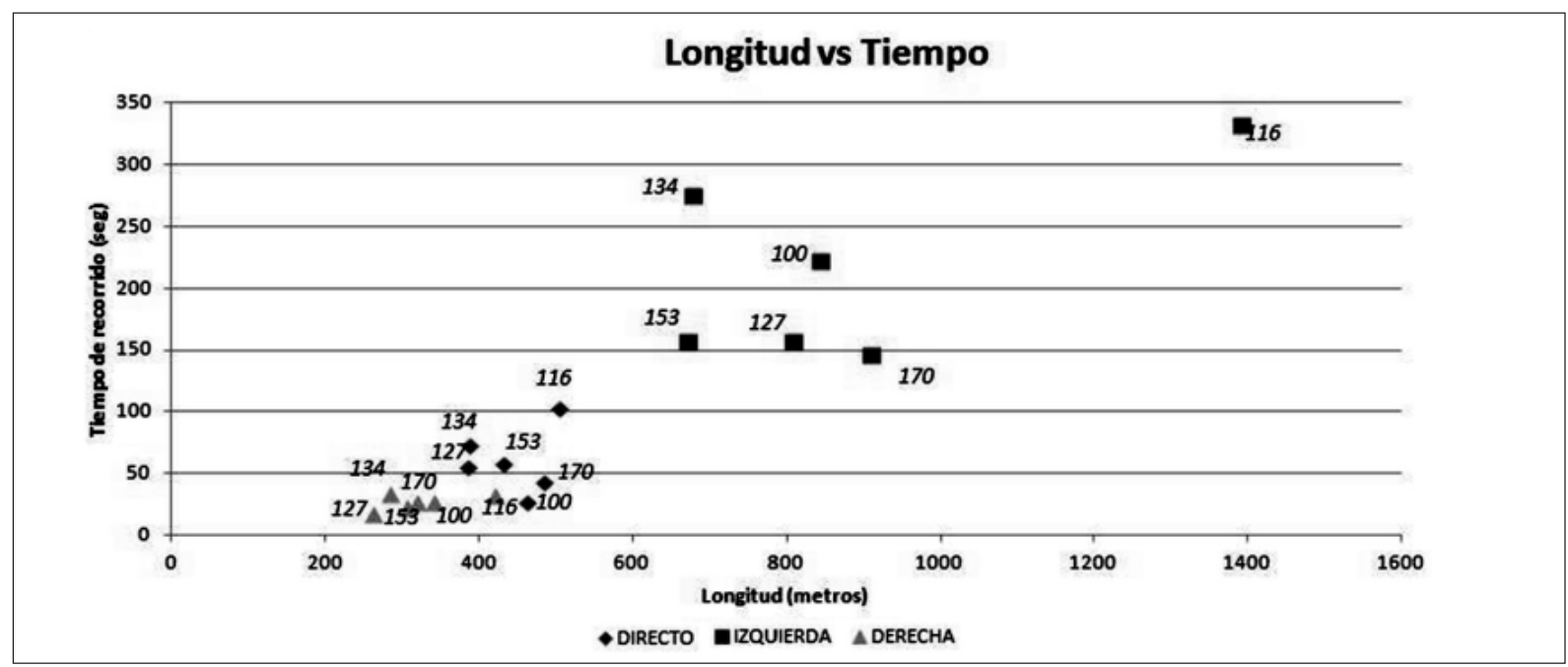

Figura 3. Longitud Vs Tiempo

Fuente: elaboración propia.

\section{RESULTADOS}

Como principal aspecto a destacar, se observa que: los giros a la derecha, en promedio, tienen un tiempo de recorrido de 25 segundos; los movimientos directos de 60 segundos, y finalmente, en los giros a la izquierda el tiempo de recorrido promedio es de 215 segundos; es decir que el tiempo de recorrido directo y el giro a la izquierda es 2,4 y 8,6 veces respectivamente del movimiento a la derecha. A pesar que los movimientos directos tienen en promedio un menor flujo vehicular que los giros a la izquierda, es más rápido realizar un movimiento directo que hacer el giro. Por lo anotado, el análisis a realizar se centra en los tiempos requeridos para recorrer los trazados de giro a izquierda en las intersecciones.

\subsection{Tiempos en movimiento a izquierda}

Tras analizar los tiempos de recorrido en los movimientos a izquierda en cada una de las intersecciones, se llegó a la conclusión de que su explicación se fundamenta en la disposición geométrica del trazado y en los flujos vehiculares ocasiona- dos por presencia de dispositivos de control o por la disposición de la actividad comercial y sus estacionamientos públicos. A continuación se presentan las situaciones diagnosticadas para cada una de las intersecciones en estudio.

En la intersección de la calle 100, los altos flujos vehiculares se deben principalmente a una intersección semafórica en el costado oriental de la Autopista Norte, a 316 metros, a partir del final del puente (figura 4).

En el caso de la intersección de la calle 116 los movimientos generados desde y hacia la Autopista Norte, en el costado occidental, se realizan mediante orejas con un trazado aproximado de 320 m (figura 5a). A su vez, la calle 116 presenta una intersección, con prioridad de pare, bajo el puente entre el flujo que va a tomar la Autopista Norte hacia el sur desde el puente de la calle 116 (oriente a sur 3'30') y quienes lo van a hacer desde la calle 116 y vienen desde el occidente (40'). Pero aún más crítica es la longitud de la oreja manzana para tomar la calle 116 en dirección occidente (cerca de $850 \mathrm{~m}$ ); la cual requiere rodear 8 calles, 


\section{investigación}

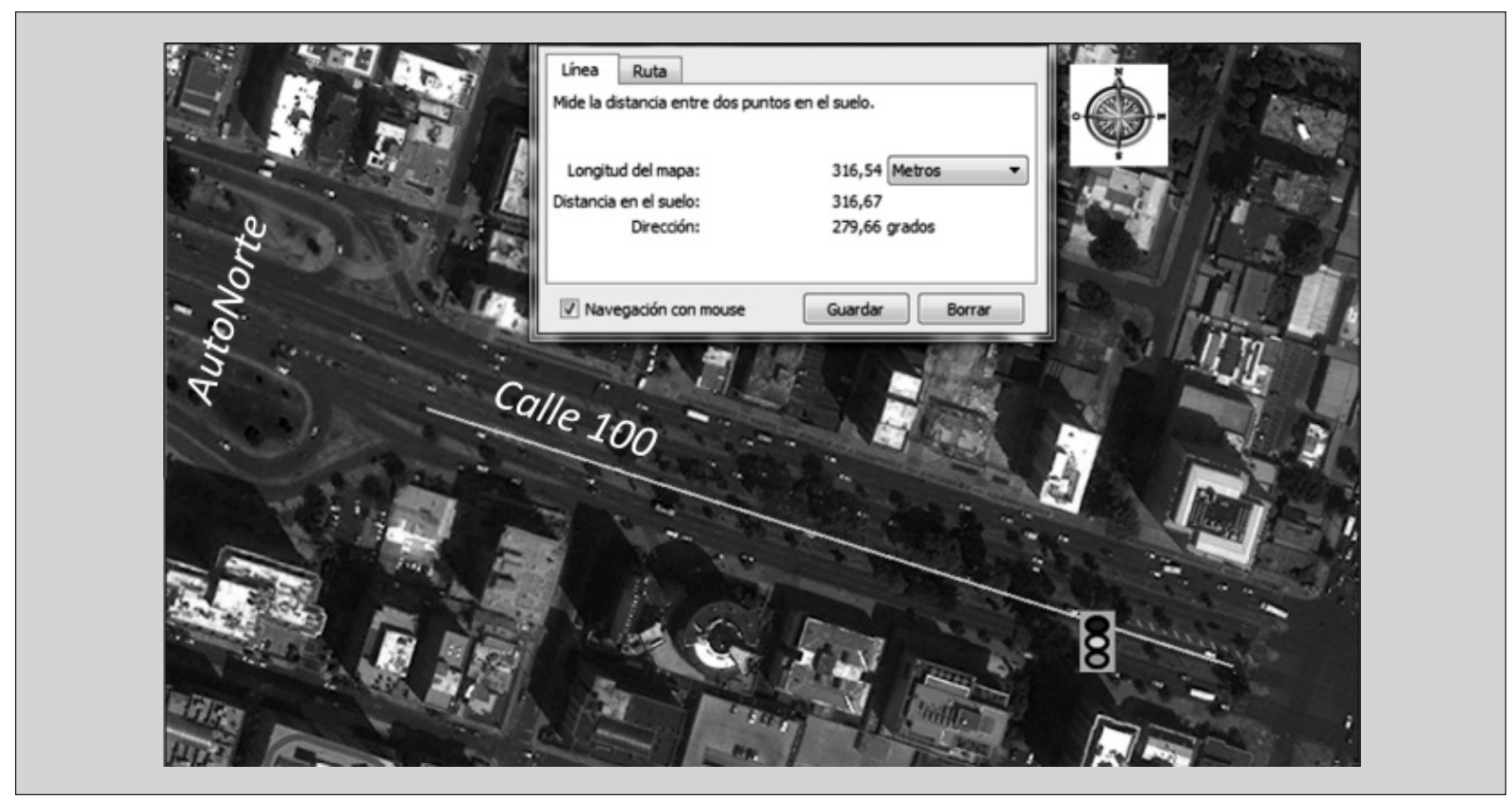

Figura 4. Ubicación de la intersección semafórica en la calle 100 al costado oriental de la Autopista Norte

Fuente: elaboración propia a partir de Google Earth

incluyendo3 intersecciones con prioridad de pare (figura 5b).

En el caso de la intersección de la calle 127, las complicaciones en el flujo vehicular se deben principalmente a que hay dos semáforos ubicados en la calle 127 a tan solo 50 metros del inicio y final de la salida y entrada de la Autopsita Norte. Estos semáforos se deben al alto flujo peatonal que se deriva de la Clínica Reina Sofía -ver el ícono del semáforo en la figura 6-. Adicionalmente, al igual que en el caso de la intersección de la calle 116, el recorrido para tomar la calle en dirección occidente desde la Autopista Norte en sentido sur a norte; la oreja manzana es de extensa longitud (cerca de $950 \mathrm{~m}$ ) y se encuentra con tres intersecciones con prioridad de pare (figura 6). La influencia del semáforo se llega a reflejar en el tránsito sobre el puente, registrando los siguientes tiempos: sur a occidente (1'08'), oriente a sur $\left(2^{\prime} 06^{\prime \prime}\right)$, occidente a oriente $\left(1^{\prime} 23^{\prime \prime}\right)$ y occidente a norte (1'13').
En el caso de la calle 170, a pesar de que la intersección tiene la mayor longitud de recorrido, el giro a izquierda hacia la calle es el que menor tiempo de recorrido registró. Este hecho se ampara en que es la intersección con menor flujo debido a su localización extrema en la mancha urbana $\mathrm{y}$, por tanto, tiene menor densidad habitacional y vehicular.

Otro caso es la oreja manzana con intersección de la calle 134. Allí se genera congestión vehicular sobre el puente de la calle en sentido de oriente a occidente para tomar la autopista al sur, llevando a tiempos de 6"01'. Cabe resaltar que la longitud de ese movimiento es relativamente corta: 245 m., lo cual sumado al hecho de que el trayecto presenta 3 intersecciones, explica mejor el alto tiempo mencionado (figura 7).

Otro aspecto importante a considerar es la presencia de retornos ubicados sobre las vías secundarias, a tan solo 100 metros de la intersección. Esta 


\section{investigación}

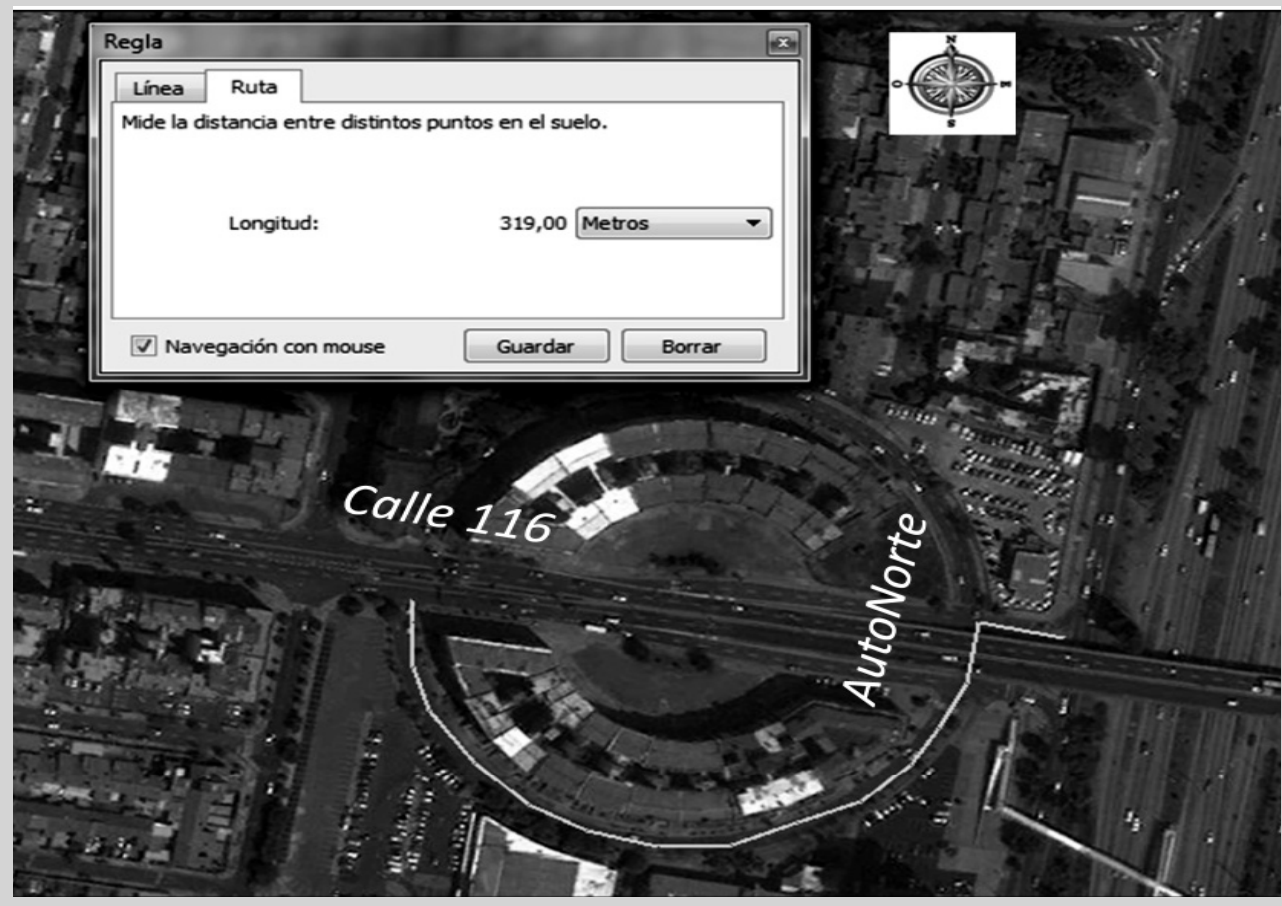

Figura 5a. Trayectos de la Autopista Norte a la calle 116, costado occidental de la autopista Fuente: elaboración propia a partir de Google Earth

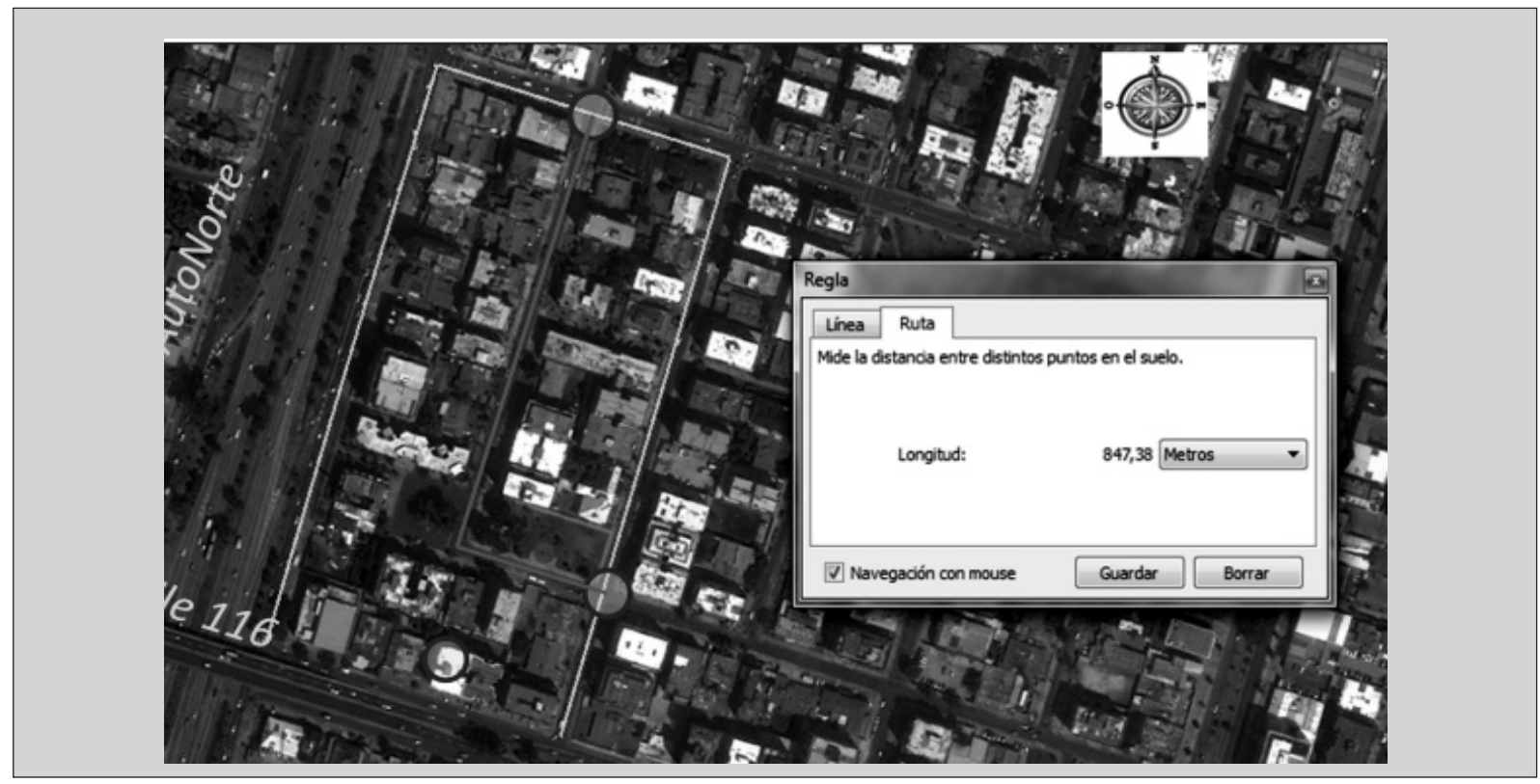

Figura 5b. Trayectos de la Autopista Norte a la calle 116 e intersecciones costado oriental Fuente: elaboración propia a partir de Google Earth 


\section{investigación}

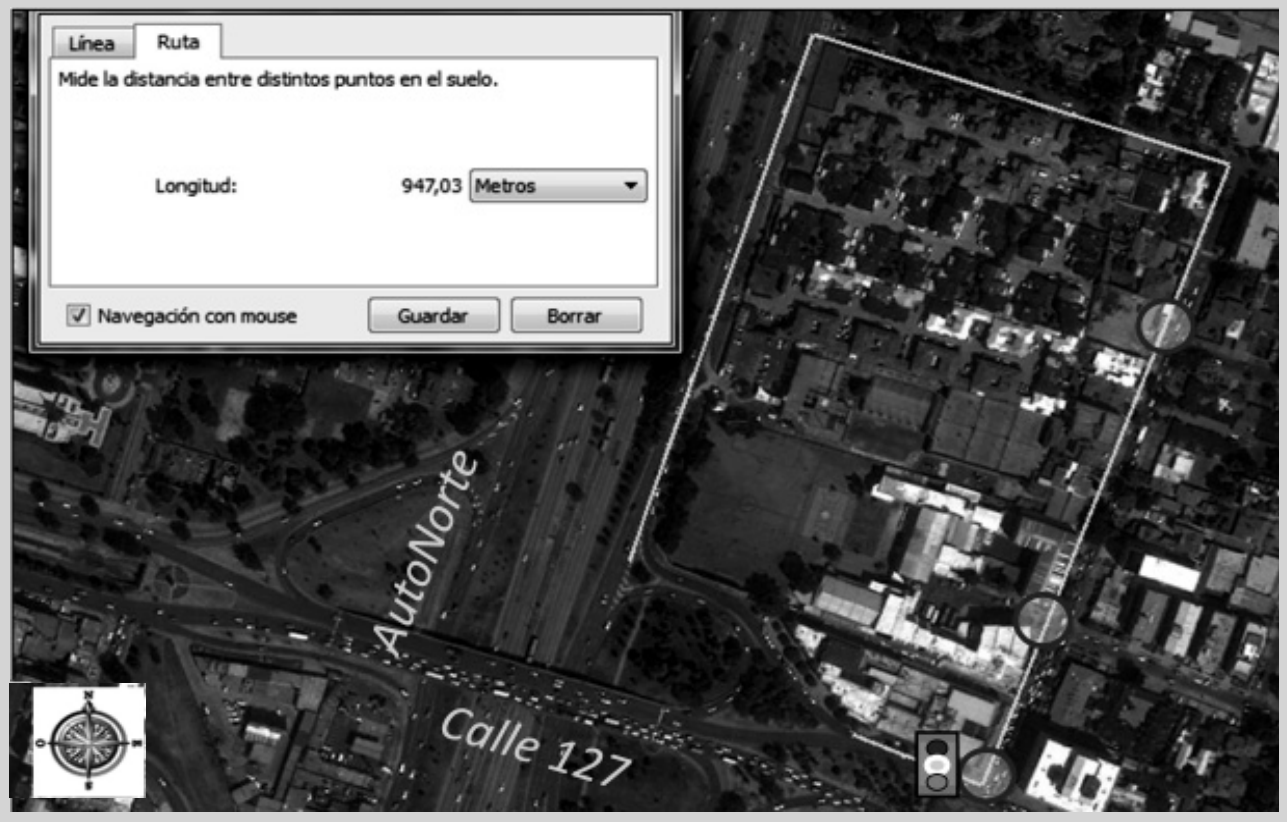

Figura 6. Trayecto desde la Autopista Norte (sur-norte) para tomar la calle 127 al occidente, y ubicación de intersecciones y semáforos

Fuente: elaboración propia a partir de Google Earth

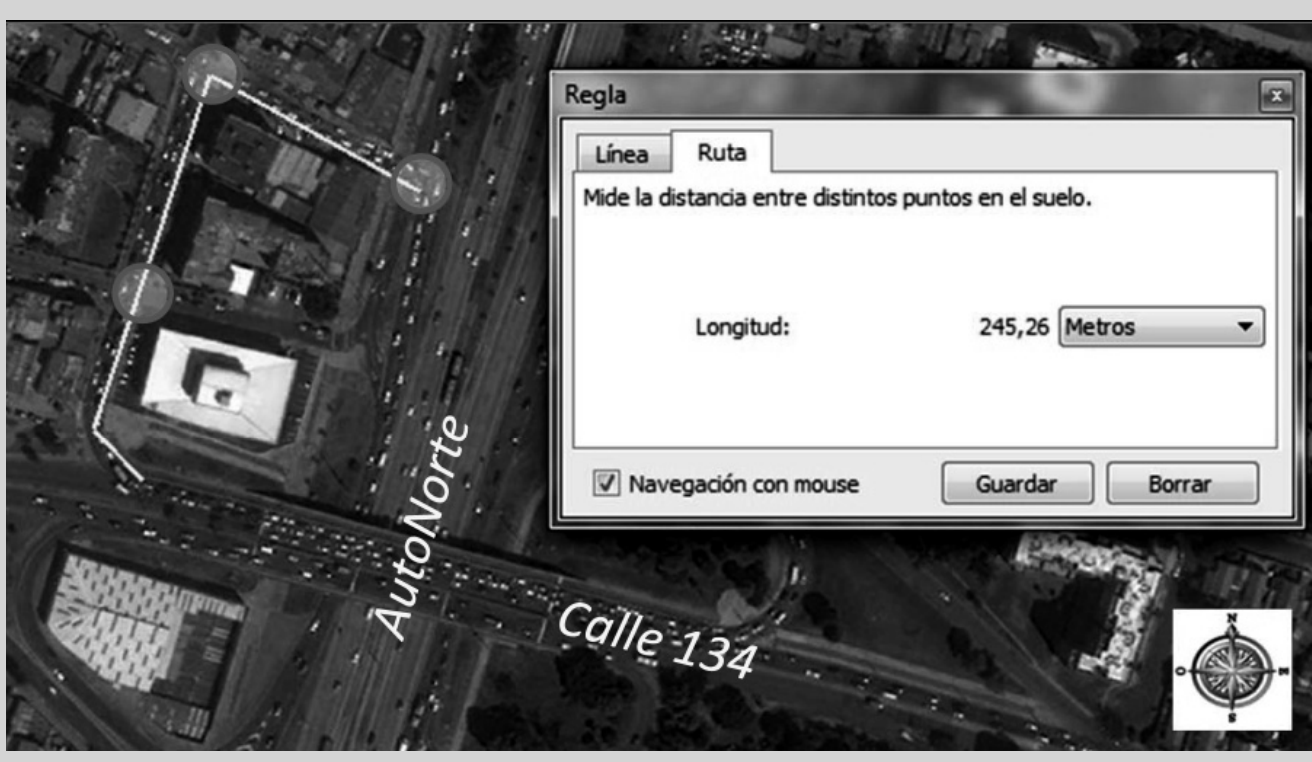

Figura 7. Trayecto de la calle 134 (oriente-occidente) para tomar la Autopista Norte en sentido sur. Con intersecciones

Fuente: elaboración propia 


\section{investigación}

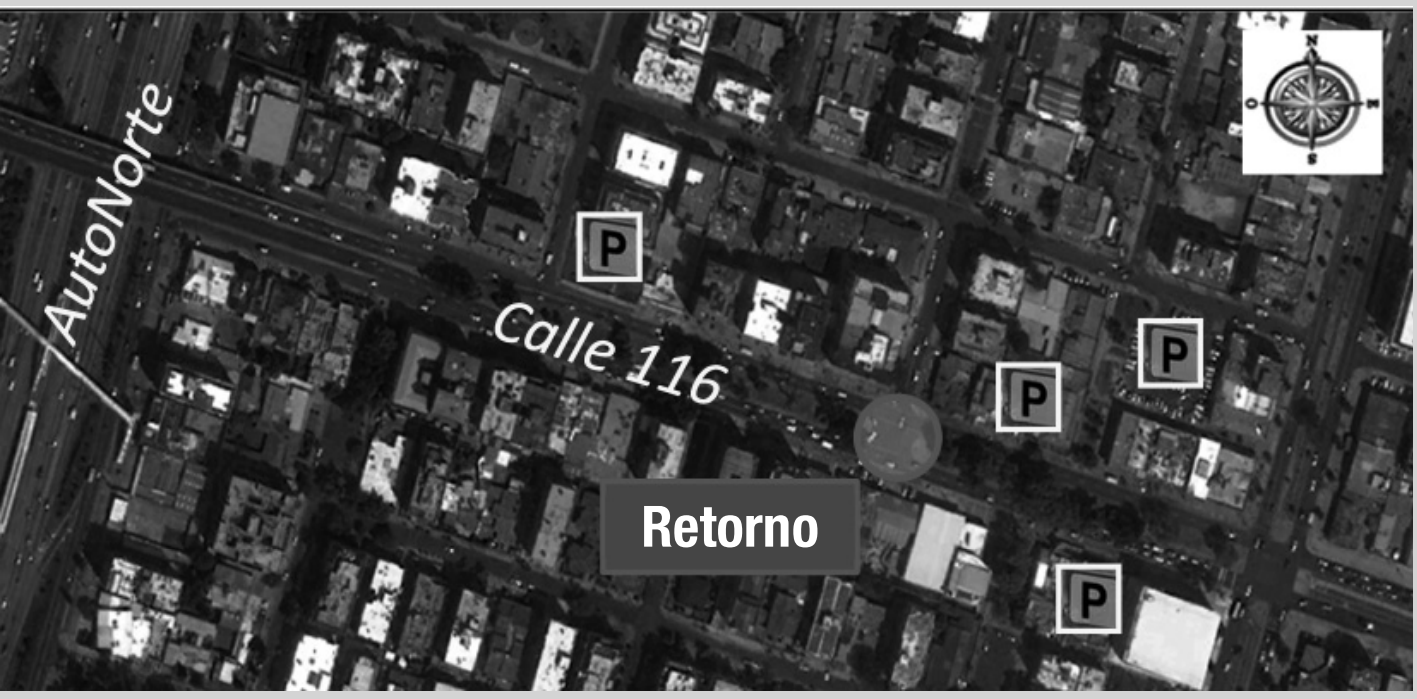

Figura 8. Ubicación de estacionamientos públicos y del retorno, en la calle 116 en su costado oriental de la Autopista Norte.

Fuente: elaboración propia

disposición genera filas de espera sobre el carril de giro. Tal es el caso de la calle 116 en su costado oriental (figura 8 y figura 9).

Por su parte, en el caso de la intersección de la calle 153, a pesar de tener un bajo flujo vehicular y relativa corta longitud de recorrido; el tiempo de recorrido es mayor de lo que se espera, debido al cuello de botella generado por la reducción de carriles 2 a 1 sobre la calle, en el puente, en dirección de oriente a occidente (figura 10).
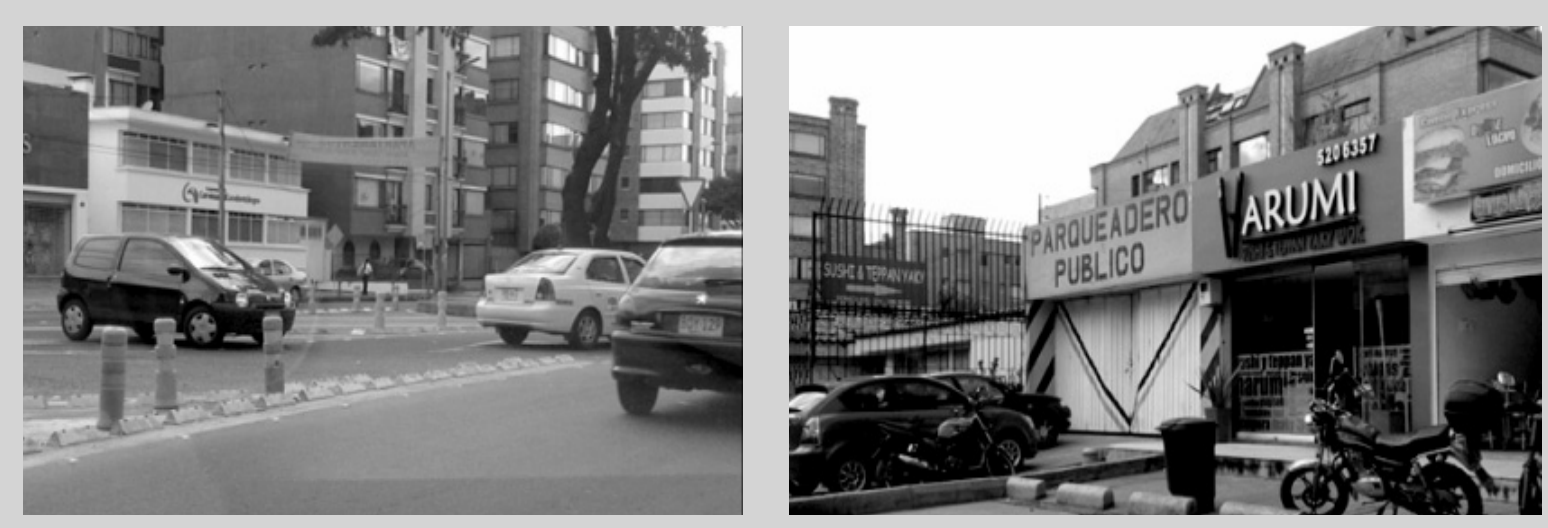

Figura 9. Calle 116. Retorno (izquierda) y oferta comercial y de estacionamiento público (derecha).

Costado oriental respecto a la Autopista Norte

Fuente: elaboración propia 


\section{investigación}

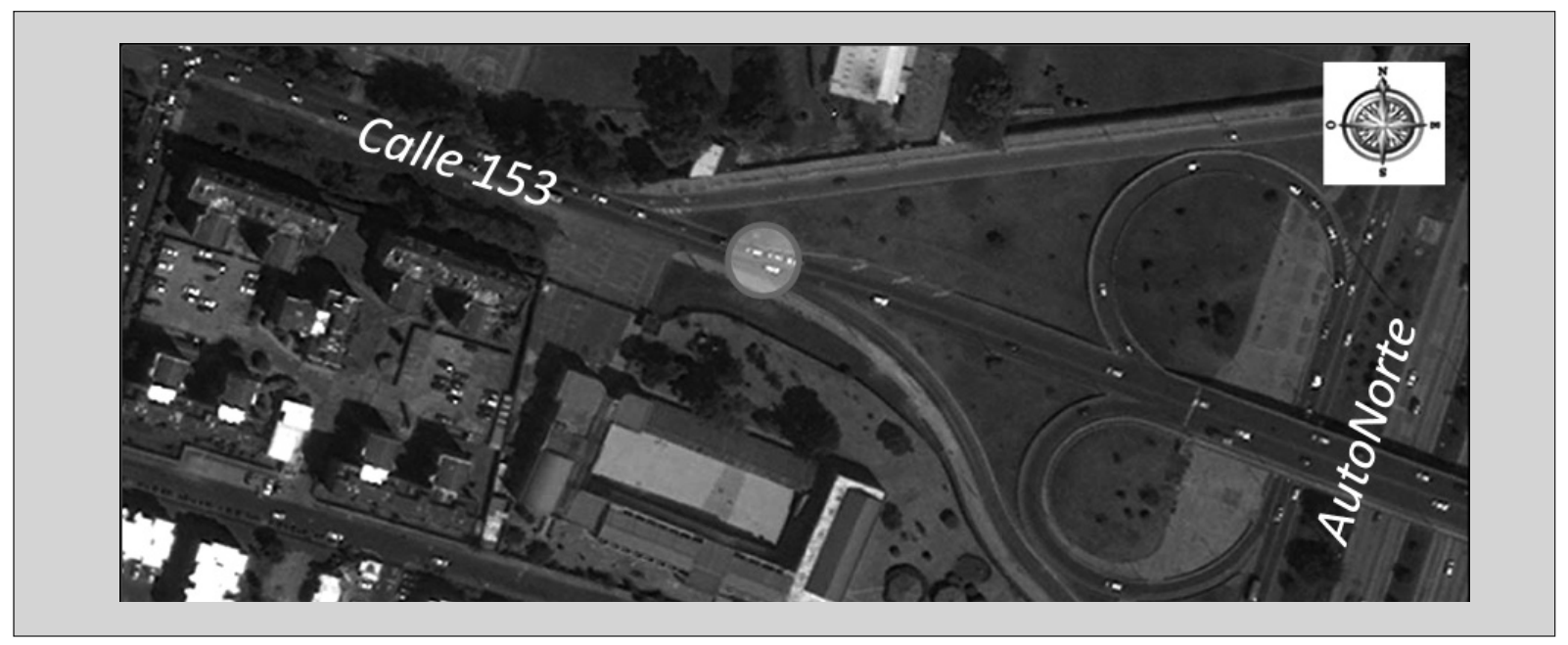

Figura 10. Calle 153. Cuello de botella (oriente a occidente) por reducción de carriles 2 a 1.

Fuente: elaboración propia

\subsection{Afectación por actividades comerciales y sus respectivos estacionamientos públicos}

A nivel de usos del suelo, la intersección de la calle 116 es un sector de actividad comercial que afecta el flujo vehicular, generando una importante oferta de estacionamientos sobre la vía (figura 9 y figura 10).
Un manejo más apropiado del uso del suelo de actividad comercial, es el que se encuentra en la calle 127. En este sector el auge comercial se presenta sobre la calle 125 (figura 11), en donde se concentra la oferta de estacionamientos públicos. Sin embargo, la calle 127 presenta una importante oferta del sector salud, que como se anotó anteriormente, contribuye a un importante flujo peatonal afectando el flujo vehicular con la pre-

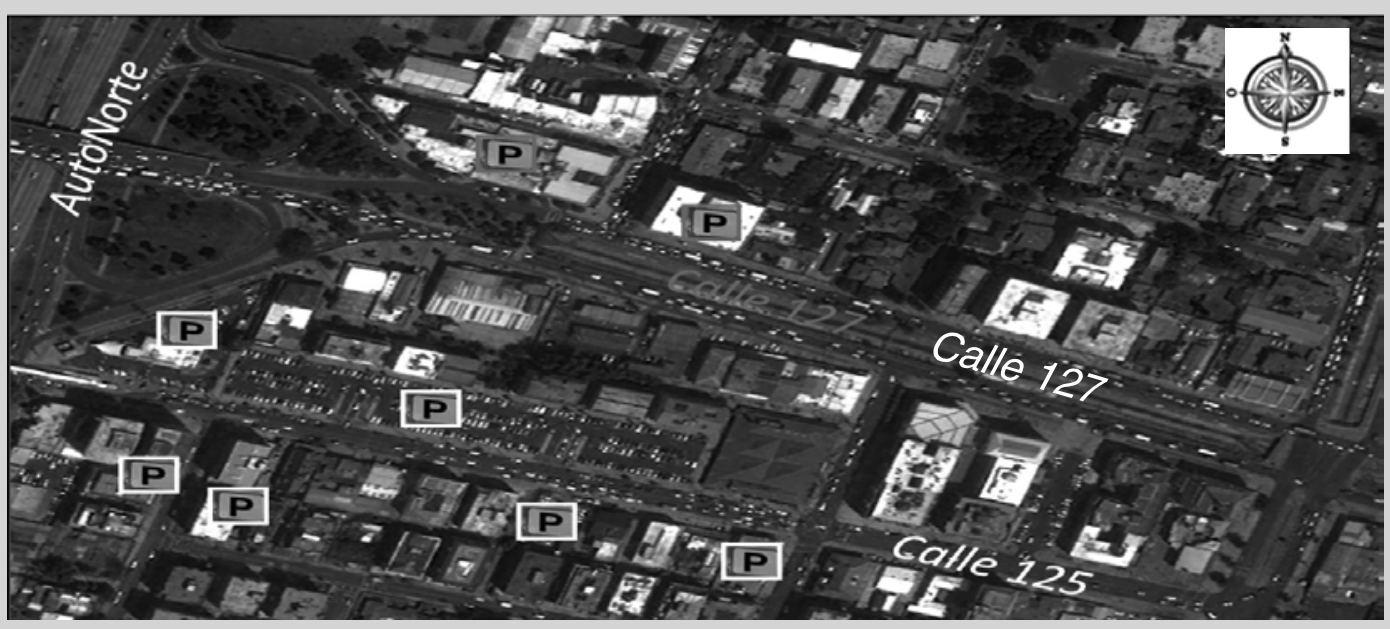

Figura 11. Concentración de la actividad comercial en la zona de la calle 127 con Autopista Norte. Oferta de parqueaderos públicos en la calle 127 y en la calle 125

Fuente: elaboración propia 


\section{investigación}

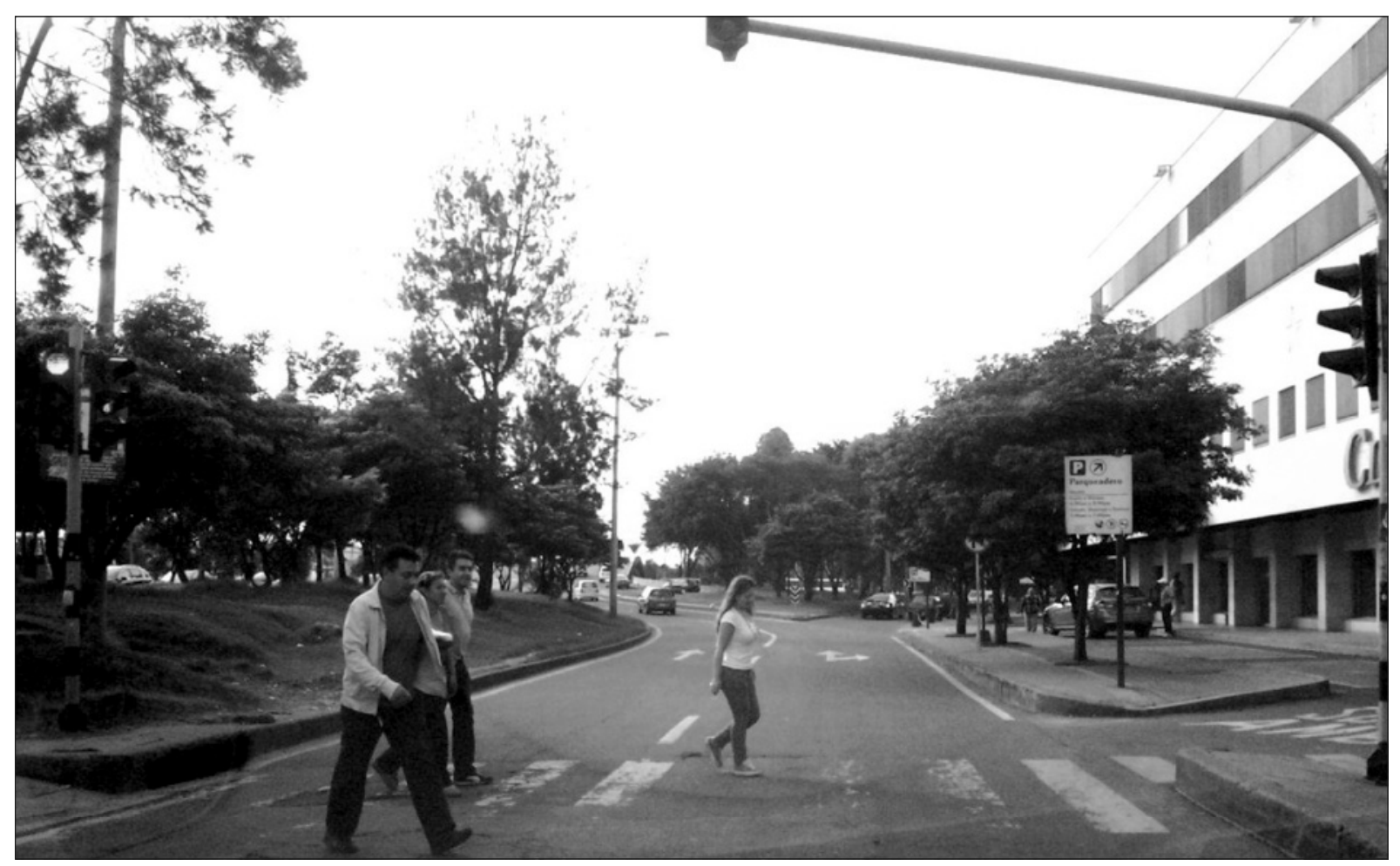

Figura 12. Semáforo peatonal en la calle 127. Costado oriente a occidente, frente a la clínica Reina Sofía Fuente: elaboración propia.

sencia de semáforos peatonales (figura 12) y con oferta de estacionamientos públicos que atienden a sus usuarios.

\section{CONCLUSIONES}

Como se ha mencionado, la geometría del trayecto de giro, la presencia de dispositivos de control, así como los estacionamientos públicos generados por la actividad comercial circundante a las vías secundarias que cruzan o intersectan un corredor de flujo continuo, como es la autopsita Norte, afectan los tiempos de giros o movimientos. Los movimientos directos y a la derecha son los menos prolongados, mientras que los movimientos a izquierda requieren más tiempo para llevarlos a cabo.

Afectaciones como: reducción de carriles, orejas manzana de considerable longitud con inter- secciones en el trayecto, dispositivos de control como semáforos y la presencia de retornos - ambos en inmediaciones de las intersecciones-, así como una importante actividad comercial y de estacionamientos sobre vías secundarias que hacen parte de la intersección; fueron los elementos determinantes de significativos tiempos para realizar los movimientos a izquierda.

Los movimientos a izquierda que requieren menor tiempo son los de la intersección de la calle 170. Si bien esta intersección es la de mayor longitud de recorrido entre las estudiadas $(911 \mathrm{~m})$, el giro a izquierda de sur a occidente presenta un tiempo de recorrido relativamente bajo: $2^{\prime} 14^{\prime \prime}$. Este hecho se ampara en que es la intersección con menor flujo debido a su localización extrema en la mancha urbana y, por tanto, con menor densidad habitacional y vehicular. 
Luego, siguen en orden de menor tiempo para el movimiento a izquierda las intersecciones de las calles 153 y 127 . Respecto de la primera intersección, a pesar de registrar una corta longitud de recorrido promedio respecto a las demás $(648 \mathrm{~m})$, el tiempo requerido es elevado (5'08' $)$, debido al cuello de botella generado por la reducción de carriles 2 a 1 sobre la calle, en el puente, en dirección de oriente a occidente; hecho que propicia un flujo relativamente significativo (985 veh/hora).

Por su parte, la intersección de la calle 127 a pesar de presentar una importante longitud de recorrido en la oreja manzana del costado oriental de la autopista $(947 \mathrm{~m})$ y contar con tres intersecciones de paso prioritario y un dispositivo de control - semáforo peatonal—, registra un tiempo promedio relativamente moderado $\left(1^{\prime} 08^{\prime \prime}\right)$.

La intersección que sigue en complejidad por el tiempo requerido para realizar el movimiento a izquierda es la de la calle 100. En este caso, la circulación en el puente se ve afectada por el semáforo ubicado al costado occidental. Este semáforo genera fenómeno de filas de espera que implican una tasa de flujo de 812 vehículos/hora, lo cual lleva al tiempo promedio de giro de occidente al norte de 5'28'". En el costado occidental del puente hay otro semáforo a sólo $250 \mathrm{~m}$ de distancia desde el final del puente. Esto hace que los movimientos de oriente a sur lleguen a valores de $4^{\prime} 11^{\prime \prime}$.

Luego, si bien la calle 134 cuenta con orejas para el giro desde y hacia el puente, el trayecto desde el puente para tomar la autopista al sur es una oreja manzana con tres puntos de intersección. Ese trayecto aumenta el flujo vehicular a lo largo de tres calles, proyectando una fila de espera sobre el puente; la cual lleva a registros de giro a izquierda de oriente a sur de $6^{\prime} 01^{\prime \prime} ; \mathrm{y}$ de sur a occidente de 3'09's.

Finalmente, se tiene la intersección de la calle 116 , en la cual una importante longitud de $1578 \mathrm{~m}$ en la oreja manzana del movimiento de sur a occidente, lleva a un tiempo de 4'55', y la presencia de una importante actividad comercial y sus estacionamientos públicos, así como un retorno en cercanía de la intersección inciden en un tiempo de hasta 8'29' ' en el movimiento de occidente a norte.

Por lo mencionado, es importante que la planificación de futuras intersecciones entre vías principales y secundarias evite, en lo posible, situaciones como las descritas. Lo más recomendable son orejas en trébol y su respectivo carril de control de acceso al puente y a la vía principal de flujo continuo.

\section{REFERENCIAS}

[1] Departamento Administrativo Nacional de Estadística - DANE, (2012, diciembre), Proyección de población 2005 a 2020, [en línea]. Disponible en: www.dane.gov.co/.

[2] Concejo de Bogotá D.C., (2012, noviembre), Proyecto de acuerdo 171 de 2011, [en línea]. Disponible en: www.alcaldia-
bogota.gov.co/sisjur/normas/Normal. jsp?i=42999.

[3] Diario El Tiempo, (2 de abril de 2011), La Autopista Norte en horas pico avanza a paso de tortuga, [en línea]. Disponible en: www.eltiempo.com/archivo/documento/ CMS-9109339]. 


\section{investigación}

[4] Dirección General de Tráfico. Ministerio del interior, Gobierno de España - DGT, (2012, agosto), Intersecciones: concepto, funcionamiento y clasificación, [en línea]. Disponible en: www.dgt.es/was6/ portal/contenidos/documentos/la_dgt/ recursos_humanos_empleo/oposiciones/ TEMA_076.pdf].

[5] Randall roads improvements study, (2012, novembre), Continuous flow intersections, [on line]. Available: www.randallroad.info/ info/downloads/cfi/].

[6] Transportation Research Board - TRB, $10^{\circ}$ Access management conference and Transportation planning conference, Dal- las, Texas, USA (2012), "Intersection and interchangedesignalternatives".

[7] Caracol radio, (abril 25 de 2011), Tránsito lento en la entrada a Bogotá por la Autopista Norte, [en línea]. Disponible en www.caracol.com.co/noticias/bogota/transito-lento-en-la-entrada-a-bogota-por-laautopista-norte/20110425/nota/1459495. aspx] [Consultado enNoviembre de 2011].

[8] Diario El Nuevo Siglo, (octubre 25 de 2011), Bogotá D.C, [en línea]. Disponible en www.elnuevosiglo.com.co/articulos/102011-sos-por-la-movilidad-de-la-autopista-norte.html. 